\title{
Ablation in Atrial Fibrillation with Ventricular Pacing Results in Similar Catheter Stability and Arrhythmia Recurrence Compared to Ablation in Sinus Rhythm with Atrial Pacing
}

\author{
Matthew Dai ${ }^{1}$, Chirag Barbhaiya ${ }^{1}$, Anthony Aizer ${ }^{2}$, Douglas Holmes ${ }^{2}$, Scott Bernstein ${ }^{3}$, \\ David Park ${ }^{4}$, Edward Kogan ${ }^{1}$, Jonathan Hyde ${ }^{1}$, Michael Spinelli ${ }^{1}$, Larry Chinitz ${ }^{3}$, and Lior \\ Jankelson $^{1}$ \\ ${ }^{1}$ NYU Langone Health \\ ${ }^{2}$ New York University Medical Center \\ ${ }^{3}$ New York University School of Medicine \\ ${ }^{4} \mathrm{NYU}$ Langone Medical Center
}

May 12, 2020

\begin{abstract}
Background: Improved catheter stability is associated with decreased arrhythmia recurrence after atrial fibrillation (AF) ablation. Recently, atrial voltage mapping in AF was demonstrated to correlate better with scar as compared to mapping in sinus rhythm (SR). However, it is unknown whether ablation of persistent AF in sinus rhythm with atrial pacing or in atrial fibrillation with ventricular pacing results in differences in catheter stability or arrhythmia recurrence. Methods: We analyzed 53 consecutive patients undergoing first-time persistent AF ablation with pulmonary vein and posterior wall isolation: 27 were cardioverted, mapped, and ablated in sinus rhythm with atrial pacing, and 26 were mapped and ablated in AF with ventricular pacing. Ablation data was extracted from the mapping system and analyzed using custom MATLAB software to determine high-frequency $(60 \mathrm{~Hz})$ catheter excursion as a novel metric for catheter spatial stability. Results: There was no difference in catheter stability as assessed by maximal catheter excursion, mean catheter excursion, or contact force variability between the atrial-paced and ventricular-paced patients. Ventricular-paced patients did have significantly greater mean contact forces compared to atrial-paced patients. One year arrhythmia-free survival was similar between the atrial paced and ventricular paced patients $(78 \%$ vs $67 \%, \mathrm{p}=0.31$ ). Conclusion: For patients with persistent AF, ablation in AF with ventricular pacing results in similar catheter stability and arrhythmia recurrence as compared to cardioversion and ablation in sinus rhythm with atrial pacing. Given the improved fidelity of mapping in AF, mapping and ablating during AF with ventricular pacing may be preferred.
\end{abstract}

\section{Background}

Radiofrequency (RF) ablation is a widely accepted and effective therapy in the management of atrial fibrillation $(\mathrm{AF})^{1-3}$. However, ablation of persistent atrial fibrillation remains a therapeutic challenge, with recurrence rates of $30-60 \%$ at one year, and a frequent need for repeat ablation. ${ }^{4-7}$ Improved catheter stability results in more consistent catheter-tissue contact, both allowing for more effective transmural lesion formation and preventing excessive force that could result in cardiac perforation. Furthermore, improved catheter stability has been shown to be associated with decreased arrhythmia recurrence following AF ablation. ${ }^{8}$ Multiple strategies and techniques exist for improving catheter stability, including high frequency jet ventilation ${ }^{9-11}$, steerable catheter sheaths ${ }^{11-13}$, and rapid atrial pacing ${ }^{14}$. However, catheter stability may be affected by the underlying atrial rhythm. In patients with persistent AF, achieving stable sinus rhythm and reliable atrial pacing may be challenging prior to ablation, and pacing can only be performed in the ventricle. It is unknown 
whether ablation in AF with ventricular pacing versus in sinus rhythm (SR) with atrial pacing has any effects on catheter stability, lesion quality, or clinical outcomes. In the present study, we sought to compare the ablation characteristics and clinical outcomes between patients with persistent AF who were mapped and ablated in sinus rhythm with atrial pacing compared to atrial fibrillation with ventricular pacing.

\section{Methods}

\section{Patient population}

We identified 53 consecutive patients at a single experienced electrophysiology center who underwent first time RF ablation for persistent AF. Of these, 27 were cardioverted then mapped and ablated in sinus rhythm with atrial pacing, and 26 were mapped and ablated in AF with ventricular pacing. Pacing was performed at a cycle length of 500-600ms, regardless of pacing site. All procedures were performed under general anesthesia with high frequency jet ventilation. Electroanatomical mapping was performed using either the circular Lasso ${ }^{\circledR}$ or five-spine PentaRay ${ }^{\circledR}$ mapping catheter and the CARTO3 ${ }^{\circledR}$ mapping system, version 4 (Biosense Webster Inc., USA). Radiofrequency ablation was performed using the ThermoCool SmartTouch ${ }^{\circledR}$ force-sensing catheter (Biosense Webster Inc., USA) using point-by-point ablation at a power of 50W. All patients underwent pulmonary vein isolation (PVI) via wide antral circumferential ablation of the left and right pulmonary veins, as well as posterior wall isolation (PWI) via superior and inferior posterior wall lines connecting the PVI lesion sets. Additional ablation of the left and right carinas and/or residual electro-active areas within the posterior wall were performed at the discretion of the operator. VisiTag lesion stability settings were set to $2 \mathrm{~mm}$ and $5 \mathrm{~s}$. Electrical isolation, including entrance and exit block, was confirmed using differential pacing and adenosine administration.

\section{Ablation analysis and catheter spatial stability}

Our group had recently described a method for precise analysis of catheter-tip spatial stability, utilizing custom developed MATLAB script applied on X-Y-Z axis tip position data acquired at $60 \mathrm{~Hz}^{8}$. Briefly, ablation data was extracted from CARTO ${ }^{\circledR}$ and analyzed using custom MATLAB script (Mathworks, USA). For each ablation lesion, the mean 3-dimensional (3-D) catheter position was calculated, and catheter excursion was defined as the distance between the position of the catheter tip, sampled at $60 \mathrm{~Hz}$, to the mean $3-\mathrm{D}$ catheter position for the lesion. The mean and maximal catheter excursion were then calculated for each lesion (see Figure 1). Contact force variability was defined as the standard deviation of the contact force for the lesion.

\section{Ablation and clinical endpoints}

The primary ablation endpoint was catheter stability, defined by mean and maximal catheter excursion. The secondary clinical endpoint was one year freedom from AF recurrence, following a 3-month blanking period. Recurrence was defined as an atrial arrhythmia lasting longer than 30 seconds on ambulatory monitoring, or an atrial arrhythmia documented on a standard 12 lead electrocardiogram. Recurrences within 3 months were included if they necessitated a repeat ablation.

\section{Statistics}

All statistical analysis was performed using SPSS Statistics 26 (IBM Corp., USA) and graphs were constructed using Prism 8 (GraphPad Software Inc., USA). Continuous variables are expressed as mean \pm standard deviation, and categorical variables are expressed as percentages. Normality of data samples was assessed using Shapiro-Wilk test. Two sample hypothesis testing was performed using either Student'st -test if samples had normal distributions or Mann-Whitney $U$ test if samples did not have normal distributions. Hypothesis testing for categorical variables was performed using Fisher's exact test. For Kaplan-Meier survival analysis, significance testing between groups was performed using log-rank test.

All patients provided written informed consent for their procedures, and all data collection and analysis was approved by the NYU Langone Medical Center Institutional Review Board.

\section{Results}




\section{Catheter stability and ablation characteristics}

Baseline patient characteristics are provided in Table 1. Ventricular-paced patients had significantly larger left atrial diameters $(4.9 \pm 0.6 \mathrm{~cm}$ vs $4.4 \pm 0.8 \mathrm{~cm}, \mathrm{p}=0.02)$, but similar left atrial volume indices $(41$ $\pm 15 \mathrm{~mL} / \mathrm{m}^{2}$ vs $\left.33 \pm 8 \mathrm{~mL} / \mathrm{m}^{2}, \mathrm{p}=0.14\right)$, as well as greater time since initial diagnosis of $\mathrm{AF}(4.4 \pm$ $4.7 \mathrm{yrs}$ vs $2.5 \pm 4.2 \mathrm{yrs}, \mathrm{p}=0.02$ ), compared to atrial-paced patients. Otherwise, there were no significant clinical or echocardiographic differences between the two groups. With respect to ablation characteristics (Table 2), there were no significant differences in measures of catheter stability between the atrial-paced and ventricular-paced patients. Atrial-paced patients had similar mean catheter excursions $(0.79 \pm 0.15 \mathrm{~mm}$ vs $0.77 \pm 0.10 \mathrm{~mm}, \mathrm{p}=0.62)$, maximal catheter excursions $(2.14 \pm 0.45 \mathrm{~mm}$ vs $2.15 \pm 0.35 \mathrm{~mm}, \mathrm{p}=0.94)$, and contact force variability $(4.2 \pm 1.0 \mathrm{~g}$ vs $4.1 \pm 0.9 \mathrm{~g}, \mathrm{p}=0.58)$, compared to ventricular-paced patients (Figure 2). In addition, the two groups had similar mean absolute impedance declines $(7.7 \pm 1.5 \mathrm{ohms}$ vs 7.1 $\pm 1.7 \mathrm{ohms}, \mathrm{p}=0.15)$ and percentage impedance declines $(6.3 \pm 1.2 \%$ vs $5.8 \pm 1.2 \%, \mathrm{p}=0.12)$. Ventricularpaced patients had greater average contact forces than atrial-paced patients $(14.5 \pm 2.4 \mathrm{~g}$ vs $13.0 \pm 1.7 \mathrm{~g}, \mathrm{p}$ $=0.02$ ) as well as greater force-time-integrals (FTIs, $107 \pm 20 \mathrm{gs}$ vs $95 \pm 15 \mathrm{gs},=0.02$ ), which was driven by the greater contact forces.

\section{Procedure characteristics}

Compared to atrial-paced patients, ventricular-paced patients had a nonsignificant trend towards increased total procedure time $(169 \pm 34 \mathrm{~min}$ vs $151 \pm 36 \mathrm{~min}, \mathrm{p}=0.08)$. There was no significant difference in $\mathrm{RF}$ time ( $43 \pm 13 \mathrm{~min}$ vs $41 \pm 13 \mathrm{~min}, \mathrm{p}=0.64)$ (Table 3$)$. There no procedural complications.

\section{Arrhythmia recurrence}

48 of $53(91 \%)$ patients completed 1 year follow up, with an average follow up length of $11.5 \pm 1.9$ months. Follow up length was similar between the two groups. Overall 6 patients $(11 \%)$ had long term continuous monitoring with an implantable device (PPM, ICD, or ILR), equally distributed between groups. The remaining patients underwent 1 or 2-week ambulatory event monitoring at 3, 6, and 12 months post-procedure (average $2.3 \pm 1.1$ monitors). $87 \%$ of patients were maintained on an anti-arrhythmic medication (Amiodarone, Dronedarone, or Flecainide) for one month post-procedure, and $85 \%$ of patients were continued on long term beta-blocker and/or calcium channel blocker therapy (Table 1). One year arrhythmia-free survival was similar between the atrial-paced and ventricular-paced patients ( $78 \%$ vs $67 \%, \mathrm{p}=0.31)$ (Figure 3 ).

\section{Discussion}

In this study, we aimed to determine if pacing site and the rhythm during AF ablation affect procedural characteristics, and most importantly catheter stability. We have recently demonstrated that improved spatial catheter stability is associated with lower rates of arrhythmia recurrence after AF ablation ${ }^{8}$. Therefore, it is important to understand the interaction of the atrial rhythm during AF ablation and catheter spatial stability.

Our data suggests that catheter ablation during atrial fibrillation with ventricular pacing, compared to sinus rhythm with atrial pacing, does not result in significant differences in high-resolution catheter spatial stability and lesion quality. Despite the difference in the underlying atrial rhythm, measures of catheter stability were similar between the two groups. In fact, ventricular-paced patients had significantly greater average contact forces (and subsequently greater FTIs), suggesting maintaining adequate catheter-tissue contact may be easier during atrial fibrillation, when there is less myocardial motion. There were also no differences in impedance decline between atrial paced and ventricular paced patients.

Ventricular-paced patients had longer procedure times and trended towards a greater number of electrical cardioversions. This can be explained by the fact that some patients in this group had unsuccessful cardioversion attempts at the beginning of the procedure, which led to the decision to perform ablation in $\mathrm{AF}$ with ventricular pacing instead. All ventricular-paced patients in our study were successfully cardioverted to sinus rhythm by procedure end. 
Catheter ablation of persistent atrial fibrillation remains a therapeutic challenge, with high recurrence rates and frequent need for repeat ablations. The arrhythmogenic substrate in persistent atrial fibrillation is complex and incompletely elucidated, and although the pulmonary veins and posterior wall are frequently implicated, there is no consensus regarding optimal ablation targets or strategy ${ }^{15-19}$. Whereas the majority of ectopic foci in paroxysmal AF reside within the pulmonary vein-left atrial interface ${ }^{20,21}$, the mechanisms behind persistent AF are more complex and often the result of long-standing atrial remodeling ${ }^{22,23}$. Underlying atrial myopathy and fibrosis, particularly within the posterior wall of the left atrium, can serve as additional triggers and propagators of persistent AF. Voltage mapping can be performed to identify areas of scar and fibrosis, although previous data has shown that voltage amplitudes are affected by the underlying atrial rhythm ${ }^{24-26}$. A recent study by Qureshi et al. found that mapping during atrial fibrillation, compared to sinus rhythm, may actually be more sensitive and specific in identifying low voltage regions that correlate with atrial fibrosis on cardiac $\mathrm{MRI}^{27}$. More accurate intra-procedural voltage mapping would provide invaluable information regarding identifying arrhythmogenic substrate and refining ablation strategy.

Overall one year arrhythmia-free survival was $73 \%$, which is in line with previous data for persistent $\mathrm{AF}$ ablation $^{4,6,7}$ and there was no significant difference in recurrence between the atrial-paced and ventricularpaced patients. The ventricular-paced patients had significantly greater LA diameters, time since AF diagnosis, as well as trend toward greater LA volume indices and lower LV ejection fraction. These measures have been shown to be associated with increased arrhythmia recurrence ${ }^{28-31}$ thus sample size may have confounded recurrence outcomes.

\section{Limitations}

This was a retrospective study of a consecutive cohort with a follow up time of 1 year. The small sample size was insufficient for logistic regression analysis to assess for predictors of arrhythmia recurrence. Patients ablated in AF with ventricular pacing may represent a subset with more complex substrate, evident by resistance to DCCV.

\section{Conclusions}

Ablation of persistent atrial fibrillation in atrial fibrillation with ventricular pacing, compared to sinus rhythm with atrial pacing, results in similar catheter stability and lesion quality as assessed by impedance decline and ablation parameters. Given the recent evidence suggesting voltage mapping during atrial fibrillation may provide more accurate assessment of atrial fibrosis, it may be preferable, at least in patients presenting in $\mathrm{AF}$, to ablate during atrial fibrillation with ventricular pacing, and defer cardioversion until after procedure completion.

\section{Acknowledgements}

The authors would like to thank Mr. Morris Ziv-Ari (Biosense Webster Inc., USA) for his assistance with MATLAB scripting.

\section{References}

1. January CT, Wann LS, Alpert JS, et al. 2014 AHA/ACC/HRS guideline for the management of patients with atrial fibrillation: executive summary: a report of the American College of Cardiology/American Heart Association Task Force on practice guidelines and the Heart Rhythm Society. Circulation . 2014;130(23):20712104. doi:10.1161/CIR.0000000000000040

2. Calkins H, Hindricks G, Cappato R, et al. 2017 HRS/EHRA/ECAS/APHRS/SOLAECE expert consensus statement on catheter and surgical ablation of atrial fibrillation. Heart Rhythm . 2017;14(10):e275-e444. doi:10.1016/j.hrthm.2017.05.012

3. January CT, Wann LS, Calkins H, et al. 2019 AHA/ACC/HRS Focused Update of the 2014 AHA/ACC/HRS Guideline for the Management of Patients With Atrial Fibrillation: A Report of the American College of Cardiology/American Heart Association Task Force on Clinical Practice Guidelines and the Heart Rhythm Society. J Am Coll Cardiol . 2019;74(1):104-132. doi:10.1016/j.jacc.2019.01.011 
4. Yubing W, Yanping X, Zhiyu L, et al. Long-term outcome of radiofrequency catheter ablation for persistent atrial fibrillation. Medicine (Baltimore) . 2018;97(29). doi:10.1097/MD.0000000000011520

5. Verma A, Jiang C, Betts TR, et al. Approaches to catheter ablation for persistent atrial fibrillation. $N$ Engl J Med . 2015;372(19):1812-1822. doi:10.1056/NEJMoa1408288

6. Wynn GJ, Das M, Bonnett LJ, Panikker S, Wong T, Gupta D. Efficacy of catheter ablation for persistent atrial fibrillation: a systematic review and meta-analysis of evidence from randomized and nonrandomized controlled trials. Circ Arrhythm Electrophysiol . 2014;7(5):841-852. doi:10.1161/CIRCEP.114.001759

7. Outcomes of long-standing persistent atrial fibrillation ablation: A systematic review - Heart Rhythm. Accessed April 18, 2020. https://www.heartrhythmjournal.com/article/S1547-5271(10)00039-1/fulltext\#sec11

8. Jankelson L, Dai M, Bernstein S, et al. Quantitative analysis of ablation technique predicts arrhythmia recurrence following atrial fibrillation ablation. Am Heart J . 2020;220:176-183. doi:10.1016/j.ahj.2019.11.011

9. Goode JS, Taylor RL, Buffington CW, Klain MM, Schwartzman D. High-frequency jet ventilation: Utility in posterior left atrial catheter ablation. Heart Rhythm . 2006;3(1):13-19. doi:10.1016/j.hrthm.2005.09.013

10. Sivasambu B, Hakim JB, Barodka V, et al. Initiation of a High-Frequency Jet Ventilation Strategy for Catheter Ablation for Atrial Fibrillation: Safety and Outcomes Data. JACC Clin Electrophysiol . 2018;4(12):1519-1525. doi:10.1016/j.jacep.2018.08.016

11. Hutchinson MD, Garcia FC, Mandel JE, et al. Efforts to enhance catheter stability improve atrial fibrillation ablation outcome.Heart Rhythm . 2013;10(3):347-353. doi:10.1016/j.hrthm.2012.10.044

12. Piorkowski C, Kottkamp H, Gerds-Li J-H, et al. Steerable sheath catheter navigation for ablation of atrial fibrillation: a case-control study. Pacing Clin Electrophysiol PACE . 2008;31(7):863-873. doi:10.1111/j.15408159.2008.01101.x

13. Piorkowski C, Eitel C, Rolf S, et al. Steerable versus nonsteerable sheath technology in atrial fibrillation ablation: a prospective, randomized study. Circ Arrhythm Electrophysiol . 2011;4(2):157-165. doi:10.1161/CIRCEP.110.957761

14. Aizer A, Cheng AV, Wu PB, et al. Pacing Mediated Heart Rate Acceleration Improves Catheter Stability and Enhances Markers for Lesion Delivery in Human Atria During Atrial Fibrillation Ablation. JACC Clin Electrophysiol . 2018;4(4):483-490. doi:10.1016/j.jacep.2017.12.017

15. Katritsis D, Merchant FM, Mela T, Singh JP, Heist EK, Armoundas AA. Catheter Ablation of Atrial Fibrillation: The Search for Substrate-Driven End Points. J Am Coll Cardiol . 2010;55(21):2293-2298. doi:10.1016/j.jacc.2010.03.016

16. O’Neill MD, Jaïs P, Takahashi Y, et al. The stepwise ablation approach for chronic atrial fibrillationEvidence for a cumulative effect. J Interv Card Electrophysiol . 2006;16(3):153-167. doi:10.1007/s10840-0069045-1

17. Magnani S, Muser D, Chik W, Santangeli P. Adjunct ablation strategies for persistent atrial fibrillationbeyond pulmonary vein isolation. $J$ Thorac Dis . 2015;7(2):178-184. doi:10.3978/j.issn.2072-1439.2015.01.25

18. Kirchhof P, Calkins H. Catheter ablation in patients with persistent atrial fibrillation. Eur Heart $J$. 2017;38(1):20-26. doi:10.1093/eurheartj/ehw260

19. Roten Laurent, Derval Nicolas, Jaïs Pierre. Catheter Ablation for Persistent Atrial Fibrillation. Circ Arrhythm Electrophysiol . 2012;5(6):1224-1232. doi:10.1161/CIRCEP.112.974873

20. Haïssaguerre M, Hocini M, Sanders P, et al. Catheter ablation of long-lasting persistent atrial fibrillation: clinical outcome and mechanisms of subsequent arrhythmias. J Cardiovasc Electrophysiol . 2005;16(11):11381147. doi:10.1111/j.1540-8167.2005.00308.x 
21. Haïssaguerre M, Jaïs P, Shah DC, et al. Spontaneous initiation of atrial fibrillation by ectopic beats originating in the pulmonary veins. $N$ Engl $J$ Med . 1998;339(10):659-666. doi:10.1056/NEJM199809033391003

22. Iwasaki Yu-ki, Nishida Kunihiro, Kato Takeshi, Nattel Stanley. Atrial Fibrillation Pathophysiology. Circulation . 2011;124(20):2264-2274. doi:10.1161/CIRCULATIONAHA.111.019893

23. Lau DH, Linz D, Schotten U, Mahajan R, Sanders P, Kalman JM. Pathophysiology of Paroxysmal and Persistent Atrial Fibrillation: Rotors, Foci and Fibrosis. Heart Lung Circ . 2017;26(9):887-893. doi:10.1016/j.hlc.2017.05.119

24. Masuda M, Fujita M, Iida O, et al. Comparison of Left Atrial Voltage between Sinus Rhythm and Atrial Fibrillation in Association with Electrogram Waveform. Pacing Clin Electrophysiol . 2017;40(5):559-567. doi:10.1111/pace.13051

25. Yagishita A, Oliveira SD, Cakulev I, et al. Correlation of Left Atrial Voltage Distribution Between Sinus Rhythm and Atrial Fibrillation: Identifying Structural Remodeling by 3-D Electroanatomic Mapping Irrespective of the Rhythm. J Cardiovasc Electrophysiol . 2016;27(8):905-912. doi:10.1111/jce.13002

26. Rodríguez-Mañero M, Valderrábano M, Baluja A, et al. Validating Left Atrial Low Voltage Areas During Atrial Fibrillation and Atrial Flutter Using Multielectrode Automated Electroanatomic Mapping. JACC Clin Electrophysiol . 2018;4(12):1541-1552. doi:10.1016/j.jacep.2018.08.015

27. Qureshi NA, Kim SJ, Cantwell CD, et al. Voltage during atrial fibrillation is superior to voltage during sinus rhythm in localizing areas of delayed enhancement on magnetic resonance imaging: An assessment of the posterior left atrium in patients with persistent atrial fibrillation. Heart Rhythm . 2019;16(9):1357-1367. doi:10.1016/j.hrthm.2019.05.032

28. Kim YG, Choi J-I, Boo KY, et al. Clinical and Echocardiographic Risk Factors Predict Late Recurrence after Radiofrequency Catheter Ablation of Atrial Fibrillation. Sci Rep . 2019;9(1):1-9. doi:10.1038/s41598019-43283-7

29. Winkle RA, Jarman JWE, Mead RH, et al. Predicting atrial fibrillation ablation outcome: The CAAP-AF score. Heart Rhythm . 2016;13(11):2119-2125. doi:10.1016/j.hrthm.2016.07.018

30. Shin S-H, Park M-Y, Oh W-J, et al. Left Atrial Volume Is a Predictor of Atrial Fibrillation Recurrence After Catheter Ablation. J Am Soc Echocardiogr . 2008;21(6):697-702. doi:10.1016/j.echo.2007.10.022

31. McCready JW, Smedley T, Lambiase PD, et al. Predictors of recurrence following radiofrequency ablation for persistent atrial fibrillation.EP Eur . 2011;13(3):355-361. doi:10.1093/europace/euq434

\section{Tables and Figures}

Table 1. Baseline Clinical Characteristics

\begin{tabular}{lllll}
\hline & Overall $(\mathrm{n}=53)$ & A-paced $(\mathrm{n}=27)$ & V-paced $(\mathrm{n}=26)$ & $p$ \\
\hline Sex $(\%$ male) & $74 \%(39 / 53)$ & $74 \%(20 / 27)$ & $73 \%(19 / 26)$ & 1 \\
Age (yrs) & $63 \pm 12$ & $64 \pm 10$ & $62 \pm 14$ & 0.92 \\
BMI $\left(\mathrm{kg} / \mathrm{m}^{2}\right)$ & $30.7 \pm 6.5$ & $29.3 \pm 4.6$ & $32.1 \pm 7.8$ & 0.12 \\
LVEF $(\%)$ & $55 \pm 12$ & $57 \pm 12$ & $53 \pm 13$ & 0.11 \\
LA Diameter $(\mathrm{cm})$ & $4.6 \pm 0.7$ & $4.4 \pm 0.8$ & $4.9 \pm 0.6$ & 0.02 \\
LA Volume Index $\left(\mathrm{mL} / \mathrm{m}^{2}\right)$ & $37 \pm 13$ & $33 \pm 8$ & $41 \pm 15$ & 0.14 \\
Years Since AF Diagnosis & $3.4 \pm 4.5$ & $2.5 \pm 4.2$ & $4.4 \pm 4.7$ & 0.02 \\
$\mathrm{CHA}_{2} \mathrm{DS}_{2}$-VASc & $2.1 \pm 1.7$ & $2.0 \pm 1.6$ & $2.2 \pm 1.7$ & 0.78 \\
$\mathrm{HTN}$ & $62 \%(33 / 53)$ & $59 \%(16 / 27)$ & $65 \%(17 / 26)$ & 0.78 \\
DM & $15 \%(8 / 53)$ & $19 \%(5 / 27)$ & $12 \%(3 / 26)$ & 0.7 \\
CHF & $21 \%(11 / 53)$ & $22 \%(6 / 27)$ & $19 \%(5 / 26)$ & 1 \\
CVA & $6 \%(3 / 53)$ & $4 \%(1 / 27)$ & $8 \%(2 / 26)$ & 0.61
\end{tabular}




\begin{tabular}{lllll}
\hline & Overall $(\mathrm{n}=53)$ & A-paced $(\mathrm{n}=27)$ & V-paced $(\mathrm{n}=26)$ & $p$ \\
\hline Vascular Disease & $4 \%(2 / 53)$ & $7 \%(2 / 27)$ & $0 \%(0 / 26)$ & 0.49 \\
OSA & $36 \%(19 / 53)$ & $37 \%(10 / 27)$ & $35 \%(9 / 26)$ & 1 \\
Post-Ablation Medications & & & & \\
Anti-arrhythmic & $87 \%(46 / 53)$ & $81 \%(22 / 27)$ & $92 \%(24 / 26)$ & 0.42 \\
BB or CCB & $85 \%(45 / 53)$ & $78 \%(21 / 27)$ & $92 \%(24 / 26)$ & 0.25 \\
Post-Ablation Monitoring & & & & \\
ILR, PPM, or ICD & $11 \%(6 / 53)$ & $11 \%(3 / 27)$ & $12 \%(3 / 26)$ & 1 \\
Number of Monitors & $2.3 \pm 1.1$ & $2.4 \pm 1.0$ & $2.1 \pm 1.2$ & 0.41 \\
\hline
\end{tabular}

$\mathrm{BMI}=$ body mass index, $\mathrm{LVEF}=$ left ventricular ejection fraction, $\mathrm{LA}=$ left atrium, $\mathrm{AF}=$ atrial fibrillation, HTN $=$ hypertension, $\mathrm{DM}=$ diabetes mellitus, $\mathrm{CHF}=$ congestive heart failure, $\mathrm{CVA}=$ cerebrovascular accident, OSA = obstructive sleep apnea, $\mathrm{BB}=$ beta-blocker, $\mathrm{CCB}=$ calcium channel blocker, ILR $=$ implantable loop recorder, PPM = permanent pacemaker, ICD = implantable cardioverter-defibrillator

Table 2. Ablation Characteristics

\begin{tabular}{lllll}
\hline & Overall $(\mathrm{n}=53)$ & A-paced $(\mathrm{n}=27)$ & V-paced $(\mathrm{n}=26)$ & $p$ \\
\hline Count & $146 \pm 44$ & $136 \pm 37$ & $156 \pm 49$ & 0.10 \\
Lesion Duration (s) & $7.4 \pm 0.8$ & $7.4 \pm 0.7$ & $7.4 \pm 0.8$ & 0.53 \\
Average Force (g) & $13.7 \pm 2.2$ & $13.0 \pm 1.7$ & $14.5 \pm 2.4$ & 0.02 \\
Contact Force Variability $(\mathrm{g})$ & $4.1 \pm 0.9$ & $4.2 \pm 1.0$ & $4.1 \pm 0.9$ & 0.58 \\
Max Power (W) & $51.1 \pm 1.0$ & $51.2 \pm .4$ & $50.9 \pm 1.4$ & 0.22 \\
Base Impedance (ohms) & $120 \pm 14$ & $120 \pm 12$ & $120 \pm 16$ & 0.90 \\
Impedance Decline (ohms) & $7.4 \pm 1.6$ & $7.7 \pm 1.5$ & $7.1 \pm 1.7$ & 0.15 \\
Percent Impedance Decline & $6.0 \pm 1.2 \%$ & $6.3 \pm 1.2 \%$ & $5.8 \pm 1.2 \%$ & 0.12 \\
FTI (gs) & $101 \pm 19$ & $95 \pm 15$ & $107 \pm 20$ & 0.02 \\
Mean Excursion (mm) & $0.78 \pm 0.12$ & $0.79 \pm 0.15$ & $0.77 \pm 0.10$ & 0.62 \\
Max Excursion (mm) & $2.15 \pm 0.40$ & $2.14 \pm 0.45$ & $2.15 \pm 0.35$ & 0.94 \\
\hline
\end{tabular}

Contact force variability was defined as the standard deviation of the contact force. FTI $=$ force time integral Table 3. Procedure Characteristics

\begin{tabular}{lllll}
\hline & Overall $(\mathrm{n}=53)$ & A-paced $(\mathrm{n}=27)$ & V-paced $(\mathrm{n}=26)$ & $p$ \\
\hline RF Time (min) & $42 \pm 13$ & $41 \pm 13$ & $43 \pm 13$ & 0.64 \\
Total Procedure Time $(\min )$ & $160 \pm 36$ & $151 \pm 36$ & $169 \pm 34$ & 0.08 \\
Number of DCCVs & $1.0 \pm 0.7$ & $0.8 \pm 0.6$ & $1.2 \pm 0.7$ & $0.09 ? \dot{\imath} ?$ \\
2 DCCVs $(\%)$ & $19 \%(10 / 53)$ & $11 \%(3 / 27)$ & $27 \%(7 / 26)$ & 0.18 \\
\hline
\end{tabular}

$\mathrm{RF}=$ radiofrequency, $\mathrm{DCCV}=$ direct current cardioversion

Figure 1. Figure 1. Example ablation lesion set with excursion measures. A) For each ablation lesion, catheter position was sampled at $60 \mathrm{~Hz}$. The orange dotted trail represents all sampled positions during a single RF lesion. Mean and maximal catheter excursion were calculated for each ablation lesion, and the radius of each sphere represents the mean catheter excursion for that lesion.

B) All patients underwent pulmonary vein isolation and posterior wall isolation. Full lesion set is projected with the left atrium removed. 
Figure 2. Measures of catheter stability in atrial-paced and ventricular-paced patients. There was no significant difference in mean excursion (A), maximal excursion (B), or standard deviation of contact force (C) between the two groups. Ventricular paced patients had significantly greater average contact force, compared to atrial paced patients $(p=0.02)(\mathrm{D})$.

Figure 3. One year arrhythmia free survival. There was no significant difference in one year arrhythmia free survival between the atrial-paced and ventricular-paced patients $(78 \%$ vs $67 \%, p=0.31)$.

\section{Hosted file}

RFX.AV2 figures 5-8-2020.pptx available at https://authorea.com/users/321186/articles/450487ablation-in-atrial-fibrillation-with-ventricular-pacing-results-in-similar-catheterstability-and-arrhythmia-recurrence-compared-to-ablation-in-sinus-rhythm-with-atrialpacing 